\title{
Insuficiencia cardíaca en el mundo real. Un punto de vista italiano
}

\author{
Aldo P. Maggioni', Francesco Orso ${ }^{1,2}$, Silvia Calabria33, Elisa Rossi ${ }^{4}$, \\ Nello Martini ${ }^{5}$, integrantes del observatorio ARNO*
}

\section{Resumen}

Objetivos: las características de la población de pacientes con insuficiencia cardíaca (IC) en los estudios clínicos aleatorizados (ECA) son, generalmente, muy distintas a las de la práctica real debido a criterios específicos de elegibilidad. Estos pacientes no representan totalmente "el mundo real” de esta condición clínica. El análisis de una base de datos administrativa puede ser útil para describir mejor las características de los pacientes con IC en el mundo real.

Métodos: los datos provienen del observatorio italiano ARNO, que incluye una población de aproximadamente 2.500.000 de personas registradas en el Servicio Sanitario Nacional Italiano (SSNI). Fueron seleccionados los egresos por IC ( 1 de enero de 2008 al 31 diciembre de 2012). Describimos las características clínicas, los tratamientos, la tasa y causas de rehospitalizaciones y los costos directos para el SSNI ocasionados durante un año de seguimiento.

Resultados: de los 2.456.739 individuos incluidos en la base de datos, 54.059 (2,2\%) fueron hospitalizados por IC, 41.413 fueron dados de alta con vida con un tratamiento prescripto para su IC. La edad media fue de $78 \pm 11$ años, las mujeres fueron el 51,4\%. Solo 26,6\% fue manejado en un ámbito cardiológico. Las comorbilidades más frecuentes fueron diabetes $(30,7 \%)$, enfermedad pulmonar obstructiva crónica (EPOC) (30,5\%) y depresión (21\%). Inhibidores de la enzima convertidora de angiotensina y antagonistas de receptores de angiotensina (IECA/ARAII), betabloqueantes (BB) y antagonistas de receptor de mineralocorticoides (ARM) fueron prescriptos en 65,8\%, 49,7\% y 42,1\% respectivamente. Al menos una rehospitalización ocurrió en $56,6 \%$ de los pacientes en un año de seguimiento, el $49 \%$ de ellas fueron por causas no cardiovasculares. El costo directo por paciente fue de 11.867 euros por año, debido fundamentalmente a la hospitalización.

Conclusiones: la evidencia del mundo real provee una descripción de las características de los pacientes y los patrones de tratamiento que son muy diferentes de aquellos reportados en los ECA. Los costos del SNISS son ocasionados principalmente por las hospitalizaciones, las cuales frecuentemente se deben a causas no cardiovasculares.

Palabras clave: $\quad$ INSUFICIENCIA CARDÍACA TRATAMIENTO FARMACOLÓGICO

\section{Real world evidence of heart failure: an Italian point of view}

\section{Summary}

Aims: the population of patients with heart failure (HF) randomized in clinical trials is generally far from real practice because selected according to specific eligibility criteria. These patients do not fully represent the "real world" of this clinical condition. Analysis of an administrative database can be useful to better describe the characteristics of real world patients with $\mathrm{HF}$.

Methods: data came from the Italian ARNO Observatory including a population of nearly 2,500,000 inhabitants of the Italian National Health Service (INHS).

1. ANMCO Research Center, Florencia, Italia.

2. Azienda Ospedaliero-Universitaria Careggi, Departamento de Geriatría, Sección de Medicina Geriátrica y Cardiología, Florencia, Italia.

3. CORE, Collaborative Outcome Research, Boloña, Italia.

4. CINECA Interuniversity Consortium, Casalecchio di Reno, Boloña, Italia.

5. Drugs \& Health, Roma, Italia.

*Ver el apéndice para la lista completa de unidades y representantes locales de salud participantes.

Traducido del inglés con autorización del autor.

Correspondencia: Dr. Aldo P. Maggioni. Correo electrónico: maggioni@anmco.it centrostudi@anmco.it

Recibido Set 27, 2017; aceptado Nov 1, 2017 
Patients were selected when discharged for HF (January 1, 2008 - December 31, 2012). We described the clinical characteristics the pharmacological treatments, rate and type of re-hospitalizations and direct costs for the INHS occurring during 1 year follow-up (FU).

Results: of the 2,456,739 subjects included in the database, 54,059 (2.2\%) were hospitalized for HF: 41,413 were discharged alive and prescribed on HF treatments. Mean age was $78 \pm 11$ years, females accounted for 51.4\%. Just $26.6 \%$ were managed in a cardiology setting. The more frequent co-morbidities were diabetes $(30.7 \%)$, COPD (30.5\%) and depression (21\%). ACE-inhibitors/Angiotensin Receptor Blockers, Betablockers and Aldosterone antagonists were prescribed in $65.8,49.7$ and $42.1 \%$ of patients.

At least one re-hospitalization occurred during the 1-year follow-up in 56.6\% of patients, $49 \%$ of them were due to non-cardiovascular causes. INHS's direct cost per patient per year was $11,867 €$ mainly driven by hospitalizations.

Conclusions: real world evidence provides a description of patients' characteristics and treatment patterns that are very different from those reported by randomized clinical trials. Costs for the INHS are mainly driven by hospitalizations which are often due to non-cardiovascular reasons.

Key words: HEART FAILURE

DRUG THERAPY

\section{Contexto general}

Los pacientes con insuficiencia cardíaca (IC) constituyen un importante problema de salud pública que afecta a millones de personas en todo el mundo(1). La IC se asocia con una elevada carga de mortalidad, morbilidad y aumento de los costos sanitarios, siendo la primera causa de hospitalización en sujetos mayores de 65 años, determinando también el mayor número de días de hospitalización ${ }^{(2-4)}$.

Los ensayos clínicos aleatorizados de IC incluyen pacientes seleccionados en base a criterios de elegibilidad y, por tanto, pueden no representar completamente la población real de pacientes con esta condición clínica asistidos en la práctica clínica. Esto se debe principalmente al hecho de que los pacientes son reclutados solo por cardiólogos en entornos especializados, mientras que los pacientes con IC suelen ser manejados también por otras figuras profesionales como los médicos de medicina interna, geriatras o médicos generales.

Por tanto, con el fin de describir mejor las características demográficas y clínicas de estos pacientes en la última década se han realizado registros observacionales grandes y bien diseñados. Sin embargo, los pacientes incluidos en estos registros son, ge-

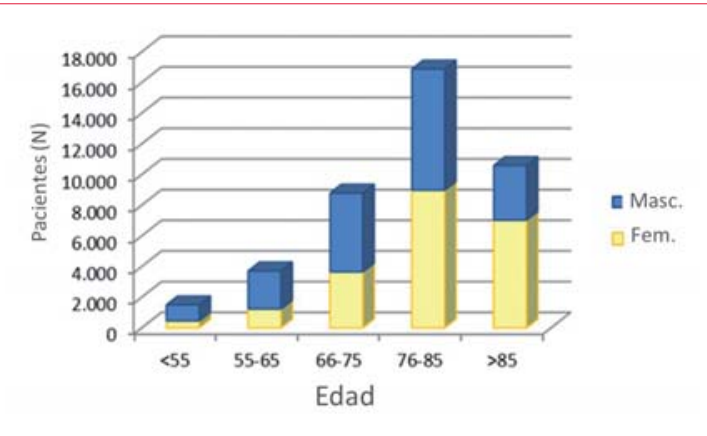

Figura 1. Distribución de los pacientes por edad y sexo. neralmente, bastante similares a los pacientes enrolados en ensayos controlados porque la gran mayoría de ellos están ingresados solo en salas de cardiología (a menudo con una alta calidad de los estándares de atención) ${ }^{(5-8)}$.

Las bases de datos administrativas han sido previamente evaluadas, específicamente en los países del norte, con el objetivo de describir la situación del "mundo real" de la IC. Sin embargo, la información sobre los tratamientos recomendados actuales para la IC, los resultados y los costos deben ser actualizados ${ }^{(9)}$.

El objetivo de la iniciativa italiana ARNO fue proporcionar una imagen más representativa de la epidemiología clínica de los pacientes con IC, analizando la base de datos local de las autoridades italianas de salud, incluyendo casi a 2.500 .000 sujetos adultos. Además, se ha realizado una evaluación de la tasa y las causas de las rehospitalizaciones y los costos debidos a IC.

\section{El marco metodológico}

Los métodos detallados se han descrito en un documento anterior ${ }^{(10)}$. Aquí se resumen las principales características del estudio $^{(10)}$.

\section{Fuente de datos}

El análisis para el estudio observacional retrospectivo se realizó desde el Observatorio ARNO (http://osservatorioarno.cineca.org), una base de datos poblacional. Desde 1987, el Observatorio ARNO recoge e integra rutinariamente los datos administrativos del Servicio Sanitario Nacional Italiano (SSNI) para cada paciente (es decir, datos demográficos de los pacientes, recetas médicas ambulatorias, descargas hospitalarias, imágenes y prescripciones de pruebas de laboratorio). Los registros de alta hospitalaria incluyen información sobre diagnósticos primarios y secunda- 


\begin{tabular}{|c|c|c|c|c|c|c|c|c|c|}
\hline & $\begin{array}{c}\text { ARNO } \\
\left(N^{\circ} 41.413\right)\end{array}$ & $\begin{array}{l}\text { ESC-HF } \\
\text { Piloto (6) } \\
\left(N^{\circ} 3.226\right)\end{array}$ & $\begin{array}{c}\text { ESC HF LT } \\
\text { (19) } \\
\text { Hospitalizados } \\
\left(N^{\circ} 5.039\right)\end{array}$ & $\begin{array}{c}\text { ESC HF LT } \\
\text { (19) } \\
\text { Ambulatorio } \\
\left(N^{\circ} 7.401\right)\end{array}$ & $\begin{array}{c}I N-H F \\
\text { Outcome }(20) \\
\left(N^{\circ} 1.855\right)\end{array}$ & $\begin{array}{c}\text { CHARM-Over } \\
\text { all (15) } \\
\left(N^{\circ} 7.601\right)\end{array}$ & $\begin{array}{c}\text { I-PRESERV } \\
E(16) \\
\left(N^{\circ} 4.128\right)\end{array}$ & $\begin{array}{l}\text { PARADIGM } \\
\text { - Trial (17) } \\
\left(N^{\circ} 8.399\right)\end{array}$ & $\begin{array}{c}\text { Relax-AHF } \\
\quad(18) \\
(n .1,161)\end{array}$ \\
\hline Tipo estudio & $\begin{array}{l}\text { Reg. } \\
\text { Adm. }\end{array}$ & $\begin{array}{c}\text { Reg. } \\
\text { Cardiol. }\end{array}$ & $\begin{array}{c}\text { Reg. } \\
\text { Cardiol. }\end{array}$ & $\begin{array}{c}\text { Reg. } \\
\text { Cardiol. }\end{array}$ & $\begin{array}{c}\text { Reg. } \\
\text { Cardiol. }\end{array}$ & ECA & ECA & ECA & ECA \\
\hline Período reclut. & $2008-2012$ & $2009-2010$ & 2011-2013 & 2011-2013 & $2007-2009$ & $1999-2001$ & $2002-2005$ & 2009-2012 & 2009-2012 \\
\hline $\begin{array}{l}\text { Tipo de IC } \\
\text { (ICFEr-ICFEp) }\end{array}$ & Todas & Todas & Todas & Todas & Todas & Todas & ICFEp & ICFEr & Todas \\
\hline \multicolumn{10}{|l|}{ Demográficos } \\
\hline Edad media (a) & 77,9 & 67 & 71 & 66 & 72 & 65,9 & 72 & 63,8 & 72 \\
\hline Femenino $(\%)$ & 51,4 & 29,7 & 37,3 & 28,8 & 39,8 & 31,5 & 60,0 & 21,9 & 37 \\
\hline \multicolumn{10}{|l|}{ Comorbilidades } \\
\hline EPOC (\%) & 30,5 & NA & 20,2 & 13,8 & 30,1 & NA & NA & $\mathrm{NA}^{*}$ & 15.8 \\
\hline $\operatorname{ERC}(\%)$ & 4,3 & 18,5 & 26,4 & 18,2 & 32,5 & NA & 30 & $\mathrm{NA}^{*}$ & NA \\
\hline $\begin{array}{l}\mathrm{TFG}<30 \\
\mathrm{ml} / \mathrm{min}\end{array}$ & $\begin{array}{l}\text { Descono- } \\
\text { cido }\end{array}$ & & 10,8 & 5,2 & 13,1 & & & & $\begin{array}{l}\text { Crit, } \\
\text { exclusión }\end{array}$ \\
\hline Cáncer (\%) & 4,6 & NA & NA & NA & NA & NA & NA & $\mathrm{NA}^{*}$ & NA \\
\hline Diabetes (\%) & 30,7 & 29,0 & 38,9 & 31,8 & 40,4 & 28,4 & 27 & 34,6 & 48.3 \\
\hline \multicolumn{10}{|l|}{ Tratamiento } \\
\hline Diuréticos (\%) & 84,2 & 82,8 & $77,3^{+}$ & 83,1 & 94,8 & 82,7 & 83 & 80,2 & 99,7 \\
\hline Digital (\%) & 26,8 & 20,6 & $26,4^{+}$ & 22,0 & 24,6 & 42,8 & 13 & 30,2 & $19,6 \%$ \\
\hline $\mathrm{BB}(\%)$ & 52,3 & 86,7 & $71, .8^{+}$ & 88,9 & 61,5 & 55,3 & 58,8 & 93,0 & 68,3 \\
\hline IECA/ARAII (\%) & 65,8 & 88,5 & $77,0^{+}$ & 89,2 & 74,5 & - & - & $100 * *$ & 70,4 \\
\hline $\mathrm{ARM}(\%)$ & 42,1 & 43,7 & $55,3^{+}$ & 59,3 & 54,7 & 16,7 & 15 & 55,6 & 31,5 \\
\hline \multicolumn{10}{|c|}{ Eventos en el seguimiento } \\
\hline $\begin{array}{l}\text { Ingresos por IC } \\
(\%) / \text { meses de } \\
\text { seguimiento(n) }\end{array}$ & $56,6 / 12$ & $31,9 / 12$ & NA & NA & $15,8 / 12$ & $22,0 / 37,7$ & $14,6 / 49,5$ & $14,2 / 27$ & NA \\
\hline $\begin{array}{l}\text { Estadía } \\
\text { hospitalaria } \\
\text { medida(días) }\end{array}$ & 12 & 8 & NA & NA & 10 & NA & NA & NA & 10 \\
\hline $\begin{array}{l}{ }^{+} \text {Al alta } \\
{ }^{*} \text { Enfermedad pulmon } \\
\text { clusión. } \\
{ }^{* *} \text { Prerandomización } \\
{ }^{\circ} \text { Criterio de inclusión } \\
{ }^{\circ} \text { ERC (TFG < } 60 \mathrm{~mL} / 1 \\
\text { I-Preserve, CHARM-O } \\
\text { (pacientes italianos inc } \\
\text { ICFEp). } \\
\text { EPOC: enfermedad pu } \\
\text { ARAII: bloqueadores d } \\
\text { suficiencia cardíaca fra }\end{array}$ & $\begin{array}{l}\text { Ir grave, ERC g } \\
\text { riterio de inclu } \\
\text { dad }>60 \text { años } \\
\text { nin/1,73m²) } \\
\text { verall (paciente } \\
\text { luidos en un re } \\
\text { monar obstruc } \\
\text { los receptores } \\
\text { cción de eyecci }\end{array}$ & $\begin{array}{l}\text { grave (TFG <3 } \\
\text { usión por proto } \\
\text { es ambulatorios } \\
\text { gistro cardioló } \\
\text { tiva crónica; E } \\
\text { s de la angioter } \\
\text { ón reducida; IC }\end{array}$ & $\begin{array}{l}30 \mathrm{ml} / \mathrm{min} / 1 \text {, } \\
\text { ocolo) } \\
\text { s con ICFEr e } \\
\text { Gico) y la escis } \\
\text { nRC: enfermed } \\
\text { nsina; ARM: a } \\
\text { CFEp: insufici }\end{array}$ & $\begin{array}{l}\text { ICFEp), Relax } \\
\text { sión de ESC H } \\
\text { lad renal cróni } \\
\text { ntagonistas de } \\
\text { encia cardíaca }\end{array}$ & $\begin{array}{l}\text { rmedades con } \\
\text { x-AHF (pacien } \\
\text { IF-LT en pacie } \\
\text { ica; IECA: inh } \\
\text { el receptor de r } \\
\text { a fracción de ey }\end{array}$ & $\begin{array}{l}\text { una esperanza } \\
\text { tes ingresados } \\
\text { ntes hospitaliz } \\
\text { ibidores de la } € \\
\text { mineralcortico } \\
\text { yección preserv }\end{array}$ & $\begin{array}{l}\text { de vida }<5 \text { añ } \\
\text { con ICFEr o I } \\
\text { zados y ambula } \\
\text { enzima de con } \\
\text { ide; IC: insufic } \\
\text { vada; TFG: tas }\end{array}$ & $\begin{array}{l}\text { ICFEp), IN-HF } \\
\text { atorios (tanto } \\
\text { versión de la a } \\
\text { ciencia cardíac } \\
\text { sa de filtración }\end{array}$ & $\begin{array}{l}\text { erios de ex- } \\
\text { Outcome } \\
\text { ICFEr como } \\
\text { ngiotensina; } \\
\text { a; ICFEr: in- } \\
\text { glomerular }\end{array}$ \\
\hline
\end{tabular}

rios. Los datos farmacéuticos derivados de la base de datos de SSNI describen las prescripciones de fármacos y consisten en el nombre del fármaco dispensado, clasificación anátomo-clínico-terapéutica (ACT), dosis, número de paquetes y fecha de dispensación. La información demográfica fue anonimizada para protección de la privacidad, según las normas italianas.

\section{Selección y seguimiento de cohortes}

De la población inicial de 2.456.739 habitantes, se seleccionó una cohorte de 54.059 pacientes hospitalizados por IC (2,2\% de la población total), desde el 1 de enero de 2008 hasta el 31 de diciembre de 2012. Se caracterizaron por, al menos, un código CM de la CIE-IX (Clasificación Internacional de Enfermeda- 
des, Novena Revisión), identificando las altas hospitalarias con diagnóstico de IC primario o secundario. Para el análisis de seguimiento a un año fueron considerados los pacientes vivos al alta ${ }^{(11)}$.

Los pacientes fueron seguidos hasta un año después de la primera alta hospitalaria, hasta el 31 de diciembre de 2013. Una pequeña proporción de pacientes podría perderse en el seguimiento debido a la migración a otras unidades locales de salud (ULS). En 2009 , solo el $6,3 \%$ de los italianos recurrieron a diferentes ULS para encontrar asistencia sanitaria más cercana a su necesidad. (http://www.ccm-network.it/ pagina.jsp?id=node/ 1633

\section{Prescripciones farmacéuticas y adherencia a largo plazo}

Las prescripciones farmacéuticas cardiovasculares (CV) y no CV se analizaron de acuerdo con las guías internacionales vigentes durante el período de recogida de datos ${ }^{(12-13)}$

Se comprobó varias veces el uso de la base de datos administrativa como una fuente confiable de datos respecto a la Continuidad de la prescripción ${ }^{(14)}$, lo cual se supone que es un indicador fiable de adherencia al tratamiento.

La continuidad de la prescripción para un tratamiento para la IC se evaluó como la relación entre el número de días de medicación suministrada dentro del intervalo de reposición y el número de días en el intervalo de reposición, con una tolerancia permitida de 20\% ( $\geq 300$ días), durante el seguimiento de un año. Esto significa que un paciente se consideró adherente si se le prescribieron 300 dosis unitarias o más durante los 365 días de seguimiento.

\section{Hospitalizaciones y causas relacionadas}

Se analizó la ocurrencia de rehospitalizaciones (\% en la cohorte total de pacientes con IC) según los siguientes indicadores: causas $\mathrm{CV}$ y no $\mathrm{CV}$ de hospitalización; días de ingreso hospitalario; mortalidad intrahospitalaria por todas las causas. La evaluación se realizó tanto para las admisiones ordinarias o las internaciones por el día y las hospitalizaciones en las diferentes salas hospitalarias (cardiología, medicina interna y otros).

\section{Costos de atención médica}

Los costos fueron evaluados durante el período de seguimiento de un año. Se evaluaron los siguientes consumos de recursos: medicamentos reembolsados en el SSNI (precios públicos reembolsados por SSNI), procedimientos diagnósticos y terapéuticos (tarifas nacionales italianas) y hospitalizaciones (tarifas nacionales italianas para el suministro de atención hospitalaria, DRG-TUC 2008). Dado que solo se consideraron los recursos directos de salud a cargo del SSNI, la perspectiva del análisis fue la del SSNI.

El costo medio se calculó por paciente y año hasta el final del seguimiento de un año o hasta que ocurrió la muerte.

\section{Características de los pacientes del mundo real frente a los de ensayos y registros de cardiología}

De los 2.456 .739 sujetos incluidos en la base de datos, $54.029(2,2 \%)$ fueron dados de alta con diagnóstico de IC. De estos pacientes, 41.413 fueron dados de alta vivos, los que conforman nuestra población de estudio. Un poco más de la cuarta parte de los pacientes fueron dados de alta desde un centro de cardiología (26,6\%); sin embargo, la mayoría de los pacientes fueron dados de alta en un servicio no cardiológico: medicina general y medicina interna fueron las salas de admisión más frecuentes $(49,7 \%$ de todas las admisiones) seguidas de salas geriátricas $(13,6 \%)$, unidades de recuperación/rehabilitación $(2,1 \%)$ y de unidades pulmonares $(1,8 \%)$.

La prevalencia de IC por edad y sexo se muestra en la figura 1. La edad media fue de $78 \pm 11$ y el sexo femenino representó el $51,4 \%$ de todos los casos. La tasa de mujeres aumentó con el aumento de la edad. La diabetes, la enfermedad pulmonar obstructiva crónica (EPOC) y la depresión fueron las comorbilidades más frecuentemente reportadas, mientras que el diagnóstico previo de cáncer y enfermedad renal crónica (ERC) se informó en menos del 5\% de los pacientes.

En la tabla 1 se presenta una comparación entre las características de los pacientes incluidos en ensayos clínicos recientes, aquellos reportados en registros cardiológicos y los analizados en este estu$\operatorname{dio}^{(6-7,75,18-20)}$.

La media de edad fue mayor en la base de datos de ARNO que en la población matriculada en los ensayos controlados, y los pacientes de sexo femenino fueron más de 50\% en el estudio ARNO, mientras que siempre fueron menores de $40 \%$ en ensayos controlados.

En tanto algunas comorbilidades, como las enfermedades renales o pulmonares crónicas severas o el cáncer fueron criterios de exclusión en los ensayos clínicos, representaron una parte relevante de los casos en la población del mundo real.

La tasa de pacientes con depresión es mayor a la informada generalmente en ensayos clínicos. Esto podría deberse al hecho de que los pacientes deprimidos generalmente están excluidos de los ensayos 
porque se consideran menos confiables para seguir los procedimientos del estudio.

Con respecto a la ERC, la baja tasa observada en nuestro estudio puede explicarse por el hecho de que se consideraron solo los pacientes ingresados previamente en el hospital por ERC, mientras que en los estudios clínicos aleatorizados (ECA) y los registros de especialidad los pacientes con altos niveles de creatinina o tasa de filtrado glomerular (TFG) bajo se incluyen en esta definición.

Nuestro estudio también muestra que el perfil clínico de los pacientes ingresados en los departamentos de medicina general/geriatría fue aún más severo que el de los pacientes ingresados en la sala de cardiología.

Las características de los pacientes incluidos en los registros cardiológicos pueden considerarse intermedias entre las observadas en los ensayos clínicos y las de los datos administrativos ${ }^{(8,9,19,20)}$.

Estos hallazgos están sustancialmente en consonancia con los informes anteriores derivados de los datos administrativos, pero la base de datos de ARNO también proporciona información sobre los resultados de los pacientes integrados con los patrones de prescripciones y los costos generales, incluyendo los procedimientos diagnósticos realizados fuera del hospital ${ }^{(9,21)}$.

Estas observaciones demuestran claramente que el traslado directo de la información derivada de un ensayo clínico a la práctica clínica diaria podría producir un impacto diferente en los resultados de los pacientes debido a las grandes diferencias no solo en las características de los pacientes, sino también en el tipo de proveedores de atención.

En otras palabras, siempre está abierta la cuestión de si un tratamiento efectivo demostrado por un ensayo puede producir un perfil similar de efectividad en la práctica real cuando los fenotipos de pacientes y profesionales de la salud son tan diferentes. Aun más complicado es el problema de la reproducibilidad del perfil de seguridad probado en ensayos en pacientes más jóvenes y sanos que en los observados en la práctica clínica.

Para alinear la práctica del mundo real y la investigación, los ensayos clínicos deben definir criterios de elegibilidad más cercanos a las características de los pacientes de la práctica clínica. Además, son necesarios "estudios de resultados" para confirmar los resultados favorables de un ensayo cuando se traducen a los pacientes de la práctica clínica. Estos estudios observacionales pos ECA podrían confirmar la eficacia/seguridad de una estrategia terapéutica en el heterogéneo contexto clínico real.
Tratamientos farmacológicos: ¿cuál es la brecha entre la práctica real y las guías?

Los diuréticos fueron la clase de fármacos más frecuentemente prescrita $(84,2 \%)$.

Entre los fármacos recomendados como clase IA por las guías de la Sociedad Europea de Cardiología (SEC) los rangos de prescripción para los inhibidores de la enzima convertidora de angiotensina y bloqueadores de los receptores de angiotensina (IECA/ARAII); betabloqueantes (BB) y antagonistas de receptor de mineralocorticoides (ARM) fueron $65,8 \%, 52,3 \%$ y $42,1 \%$ respectivamente. La digoxina se prescribió en el $26,8 \%$ de los pacientes y la ivabradina, aprobada para esta indicación en diciembre de 2012 , en $1,5 \%$ de los casos ${ }^{(12)}$.

El análisis de las dosificaciones de los fármacos más prescritos mostró que los pacientes recibieron la dosis máxima de IECA y BB recomendada por las guías solo en $15,1 \%$ y $8,5 \%$ de los casos respectivamente. La adherencia de los tratamientos también fue deficiente, de hecho, la continuidad de prescripción de los fármacos recomendados clase IA para los pacientes con IC, específicamente BB e IECA, se observó en $39,9 \%$ y 43,9\% de los pacientes respectivamente.

Si bien en nuestro estudio no es posible una evaluación precisa de la tasa real de subtratamiento debido a la falta de disponibilidad de los valores de la fracción de eyección del ventrículo izquierdo (FEVI), el estudio ARNO sugiere que, en la práctica clínica, los tratamientos con recomendación IA están subprescriptos y, cuando se prescriben, las dosis diarias máximas están muy lejos de las que han demostrado ser eficaces en los $\mathrm{ECA}^{(12)}$.

La subprescripción de los tratamientos recomendados se observó específicamente en los pacientes ingresados en los departamentos de medicina general/geriatría. Nuestro estudio confirma la variabilidad en la gestión de los pacientes en diferentes hospitales y diferentes especialidades ${ }^{(22)}$.

Una lista detallada de las razones para la no prescripción de los tratamientos recomendados para la IC o para sus dosificaciones subóptimas ya se ha informado en otro lugar ${ }^{(19)}$. Los resultados aquí presentados están en línea con un estudio europeo anterior que mostró que la prescripción de los tratamientos recomendados osciló entre $12 \%$ y $64 \%$ dependiendo de la intervención farmacológi$\mathrm{ca}^{(23)}$. El perfil clínico más grave de los pacientes incluidos en este estudio también podría explicar el bajo nivel de adecuación de la prescripción ${ }^{(19,23)}$. Dado que la falta de adecuación de las recetas a las recomendaciones de las guías podría tener un impacto negativo en los resultados de los pacientes, así como mayores costos de atención de la salud, 


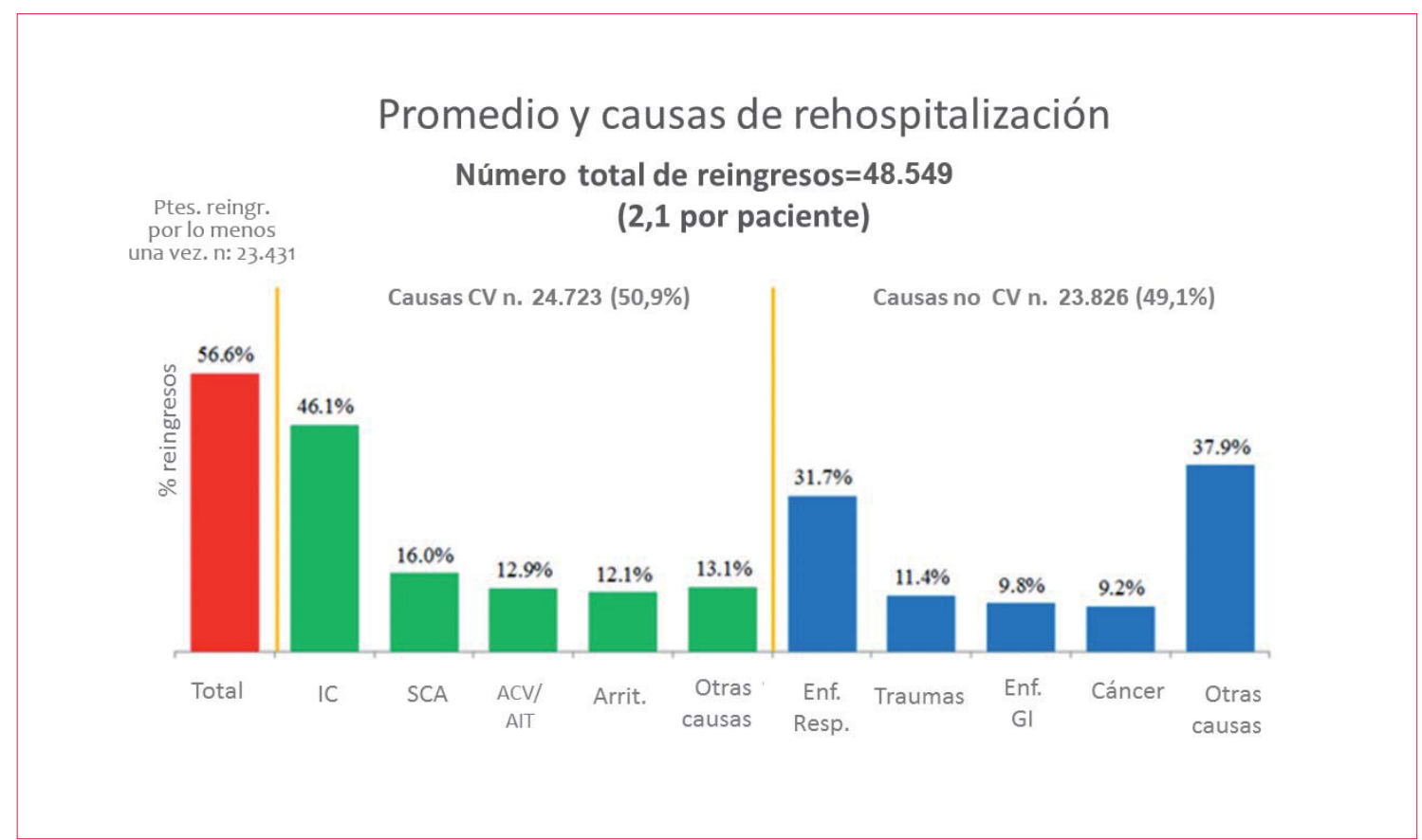

Figura 2. Tasas y causas de reingresos hospitalarios.

IC: insuficiencia cardíaca; SCA: síndrome coronario agudo; ACV/AIT: accidente cerebrovascular/accidente isquémico transitorio; CV: cardiovascular; GI: gastrointestinal.

principalmente a través de posibles ingresos hospitalarios prevenibles, se deberían hacer más esfuerzos en la planificación de programas educativos para implementar mejor las directrices en los diferentes países y a través de los diferentes entornos de atención. Esta comprensión podría ayudar a los responsables de la toma de decisiones a diseñar políticas más efectivas que en última instancia podrían ayudar a los proveedores en su búsqueda de mejorar la calidad de la atención de la salud y los resultados de los pacientes, así como potencialmente reducir los costos.

\section{El gran problema: la tasa de rehospitalización}

Durante el seguimiento de un año se registraron un total de 48.548 rehospitalizaciones (figura 2). En el $56,6 \%$ de los pacientes se realizó, al menos, una nueva hospitalización ordinaria: $51 \%$ de estos reingresos hospitalarios se debieron a causas CV y $49 \%$ a causas no CV. Entre los pacientes egresados por causas CV, la IC fue el diagnóstico principal del $45,9 \%$, mientras que síndrome coronario agudo, accidente isquémico transitorio/accidente cerebrovascular y arritmias, fueron otras causas CV en $16,1 \%, 12,9 \%$ y $12,1 \%$ de los casos. Las enfermedades respiratorias, los traumas, las enfermedades gastrointestinales y renales fueron las causas no CV más frecuentes de la hospitalización. La duración media de la hospitalización fue de 13 días. Las hospitalizaciones por IC tuvieron una duración media de 12 días.

Esta vulnerabilidad hacia una diversidad de enfermedades puede explicar por qué las intervenciones para prevenirlas deben ser realizadas por un equipo multidisciplinario. Se ha demostrado que una estrategia multidisciplinaria de intervención tiene más probabilidades de reducir los reingresos, especialmente en el área clínica de $\mathrm{IC}^{(24)}$.

Jack y colaboradores ${ }^{(25)}$ demostraron que la educación del paciente, la coordinación de la atención y la confirmación de un plan específico de medicación por parte de enfermeras registradas capacitadas más el logro de acuerdos, la educación sobre medicación y la evaluación de la adherencia por un farmacéutico clínico, condujeron a reducciones en las visitas al departamento de urgencias y en los rein$\operatorname{gresos}^{(25)}$.

\section{Los costos del Servicio Sanitario Nacional Italiano}

Durante el seguimiento de un año, un paciente con IC determinó un costo directo para el SSNI de $11.864 €$ (7.426 € si se excluyó la primera hospitalización). Analizando los componentes de este gasto durante el seguimiento de un año, el costo principal 


\section{Resumen conceptual *}

- Los registros por datos administrativos son una herramienta muy útil en el seguimiento de patologías crónicas como la insuficiencia cardíaca.

- La información de este tipo de estudio permite analizar la realidad que no muestran los estudios controlados.

- La tasa de prescripción de los fármacos que sugieren las guías, así como la dosis objetivo, en este registro han sido inferiores a lo esperado.

- Las rehospitalizaciones por causa no cardiovascular son muy frecuentes en los pacientes con IC.
- Los costos de la IC para el sistema de salud son muy altos debido principalmente a la hospitalización.

- Se deben hacer esfuerzos para lograr la aplicación de las recomendaciones de las guías de práctica clínica por parte de los proveedores sanitarios.

- Las estrategias multidisciplinarias pueden ser muy útiles para prevenir las rehospitalizaciones.

* Realizado por editor invitado debe atribuirse a las hospitalizaciones (76\%; 5.621 $€)$, seguido de las recetas de medicamentos (16\%, $1.177 €$ ) y de los exámenes de especialista/procedimientos diagnósticos $(8,5 \%, 629 €)$.

Globalmente, la IC impuso una enorme carga económica. Con el envejecimiento, la rápida expansión y la industrialización de la población mundial este valor podría seguir aumentando ${ }^{(26,27)}$.

Los costos anuales promedio observados en el presente estudio son muy altos, sobre todo en los pacientes ingresados en la sala de cardiología, aunque en general inferiores a los publicados en Estados Unidos, probablemente debido a los diferentes escenarios de atención de la salud (modalidad de cobertura de salud), valor de reembolso por hospitalizaciones, precios de las drogas y perspectiva del análisis. Al igual que en todas las evaluaciones realizadas en diferentes países, los costos de admisión hospitalaria fueron el principal determinante de los costos totales para el manejo de esta condición clínica. Curiosamente, se observaron proporciones similares de costos hace más de 25 años, lo que demuestra que no se produjeron cambios relevantes en las últimas décadas ${ }^{(28)}$.

Dado el mal pronóstico de muchos pacientes y los altos costos de la atención médica asociados a la reinternación hospitalaria, incluso pequeñas mejoras en la atención de pacientes con IC global podrían tener un impacto sustancial en la calidad de vida del paciente y el costo de la atención médi$\mathrm{ca}^{(27)}$.

Estas consideraciones son pertinentes, ya que una mayor adecuación de las recetas a las recomendaciones de las guías podría desempeñar un papel vital en la reducción de los costos de la atención de la salud y en el aumento de la eficiencia.

\section{Conclusiones}

Algunas ventajas del uso de datos administrativos son la amplitud de la cobertura de la población, la capacidad de realizar un seguimiento a largo plazo de la prescripción de medicamentos y la hospitalización. Además, este tipo de estudios proporciona información importante sobre la práctica diaria en una población "real", en nuestro caso, pacientes "reales" con IC.

Por último, la evidencia en el mundo real proporciona hallazgos que son, en gran medida, diferentes de los derivados de ECA realizados generalmente por cardiólogos. La tasa de uso de los tratamientos recomendados por las guías de práctica actuales parece estar lejos de ser óptima, así como las prescripciones de las dosis recomendadas y, por tanto, las iniciativas destinadas a aumentar la adhesión de los médicos a las guías actuales deben ser fuertemente alentadas en las distintas áreas de atención.

Las rehospitalizaciones son muy frecuentes y en casi la mitad de los casos se deben a razones no $\mathrm{CV}$, documentando el papel relevante de la edad avanzada y las comorbilidades. Los costos para el SSNI son muy altos y se deben principalmente a los costos hospitalarios. Las estrategias multidisciplinarias deben ser evaluadas para prevenir las diferentes causas de rehospitalizaciones en pacientes con IC.

\section{Agradecimientos}

Este trabajo fue apoyado parcialmente por una concesión sin restricciones de Novartis Pharma Italia. 


\section{Conflicto de intereses}

APM recibió subvenciones para la participación en comités de estudios apoyados por Bayer, Cardiorentis, Novartis. FO, SC, ER y NM no tienen nada que declarar.

\section{Bibliografía}

1. Bui AL, Horwich TB, Fonarow GC. Epidemiology and risk profile of heart failure. Nat Rev Cardiol 2011; 8(1):30-41.

2. Jhund PS, Macintyre K, Simpson CR, Lewsey JD, Stewart S, Redpath A, et al. Long-term trends in first hospitalization for heart failure and subsequent survival between 1986 and 2003: a population study of 5.1 million people. Circulation 2009; $119(4): 515-23$.

3. Roger VL. Epidemiology of heart failure. Circ Res 2013; 113(6):646-59.

4. Mozaffarian D, Benjamin EJ, Go AS, Arnett DK, Blaha MJ, Cushman M, et al. Heart disease and stroke statistics 2015 update: a report from the American Heart Association. Circulation 2015; 131(4):e29-322.

5. Abraham WT, Fonarow GC, Albert NM, Stough WG, Gheorghiade M, Greenberg BH, et al. Predictors of in-hospital mortality in patients hospitalized for heart failure: insights from the Organized Program to Initiate Lifesaving Treatment in Hospitalized Patients with Heart Failure (OPTIMIZEHF). J Am Coll Cardiol 2008; 52(5): 347-56.

6. Maggioni AP, Dahlström U, Filippatos G, Chioncel O, Crespo Leiro M, Drozdz J, et al. EURObservational Research Programme: regional differences and 1-year follow-up results of the Heart Failure Pilot Survey (ESC-HF Pilot). Eur J Heart Fail 2013; 15(7):808-17.

7. Crespo-Leiro MG, Anker SD, Maggioni AP, Coats AJ, Filippatos G, Ruschitzka F, et al. European Society of Cardiology Heart Failure Long-Term Registry (ESC-HF-LT): 1-year follow-up outcomes and differences across regions. Eur J Heart Fail 2016; 18(6):613-25.

8. Logeart D, Isnard R, Resche-Rigon M, Seronde MF, de Groote P, Jondeau G, et al. Current aspects of the spectrum of acute heart failure syndromes in a real-life setting: the OFICA study. Eur J Heart Fail 2013; 15(4):465-76.

9. Zarrinkoub R, Wettermark B, Wändell P, Mejhert M, Szulkin R, Ljunggren G, et al. The epidemiology of heart failure, based on data for 2.1 million inhabitants in Sweden. Eur J Heart Fail 2013; 15(9):995-1002

10. Maggioni AP, Orso F, Calabria S, Rossi E, Cinconze E, Baldasseroni S, et al. The real-world evi- dence of heart failure: findings from 41413 patients of the ARNO database. Eur J Heart Fail 2016; 18(4):402-10.

11. Centers for Disease Control and Prevention. International classification of diseases, ninth revision, clinical modification (ICD-9-CM)). Washington: CDC, 1998.

12. McMurray JJ, Adamopoulos S, Anker SD, Auricchio A, Böhm M, Dickstein K, et al. ESC Guidelines for the diagnosis and treatment of acute and chronic heart failure 2012: The Task Force for the Diagnosis and Treatment of Acute and Chronic Heart Failure 2012 of the European Society of Cardiology. Developed in collaboration with the Heart Failure Association (HFA) of the ESC. Eur Heart J 2012; 33(14):1787-847.

13. Yancy CW, Jessup M, Bozkurt B, Butler J, Casey DE Jr, Drazner MH, et al. 2013 ACCF/AHA guideline for the management of heart failure: a report of the American College of Cardiology Foundation/American Heart Association Task Force on Practice Guidelines. J Am Coll Cardiol 2013; 62(16):e147-239.

14. Gini R, Schuemie MJ, Francesconi P, Lapi F, Cricelli I, Pasqua A, et al. Can Italian healthcare administrative databases be used to compare regions with respect to compliance with standards of care for chronic diseases? PLoS One 2014; 9(5):e95419.

15. Pfeffer MA, Swedberg K, Granger CB, Held P, McMurray JJ, Michelson EL, et al. Effects of candesartan on mortality and morbidity in patients with chronic heart failure: the CHARM-Overall programme. Lancet 2003; 362(9386):759-66.

16. Massie BM, Carson PE, McMurray JJ, Komajda M, McKelvie R, Zile MR, et al. Irbesartan in patients with heart failure and preserved ejection fraction. N Engl J Med 2008; 359(23):2456-67.

17. McMurray JJ, Packer M, Desai AS, Gong J, Lefkowitz MP, Rizkala AR, et al. Angiotensin-neprilysin inhibition versus enalapril in heart failure. N Engl J Med 2014; 371(11):993-1004.

18. Teerlink JR, Cotter G, Davison BA, Felker GM, Filippatos G, Greenberg BH, et al. Serelaxin, recombinant human relaxin-2, for treatment of acute heart failure (RELAX-AHF): a randomised, placebo-controlled trial. Lancet 2013; 381(9860):29-39.

19. Maggioni AP, Anker SD, Dahlström U, Filippatos G, Ponikowski P, Zannad F, et al. Are hospitalized or ambulatory patients with heart failure treated in accordance with European Society of Cardiology guidelines? Evidence from 12,440 patients of the ESC Heart Failure Long-Term Registry. Eur J Heart Fail 2013; 15(10):1173-84.

20. Oliva F, Mortara A, Cacciatore G, Chinaglia A, Di Lenarda A, Gorini M, et al. Acute heart failure patient profiles, management and in-hospital outco- 
me: results of the Italian Registry on Heart Failure Outcome. Eur J Heart Fail 2012; 14(11):1208-17.

21. Corrao G, Ghirardi A, Ibrahim B, Merlino L, Maggioni AP. Burden of new hospitalization for heart failure: a population-based investigation from Italy. Eur J Heart Fail 2014; 16(7):729-36.

22. Kociol RD, Hammill BG, Fonarow GC, Heidenreich PA, Go AS, Peterson ED, et al. Associations between use of the hospitalist model and quality of care and outcomes of older patients hospitalized for heart failure. JACC Heart Fail 2013; 1(5):445-53.

23. Komajda M, Lapuerta P, Hermans N, Gonzalez-Juanatey JR, van Veldhuisen DJ, Erdmann $\mathbf{E}$, et al. Adherence to guidelines is a predictor of outcome in chronic heart failure: the MAHLER survey. Eur Heart J 2005; 26(16):1653-9.

24. Rich MW, Beckham V, Wittenberg C, Leven CL, Freedland KE, Carney RM. A multidisciplinary intervention to prevent the readmission of elderly patients with congestive heart failure. N Engl J Med 1995; 333(18):1190-5.

25. Jack BW, Chetty VK, Anthony D, Greenwald JL, Sanchez GM, Johnson AE, et al. A reengineered hospital discharge program to decrease rehospitalization: a randomized trial. Ann Intern Med 2009; 150(3):178-87.

26. Cook C, Cole G, Asaria P, Jabbour R, Francis DP. The annual global economic burden of heart failure. Int J Cardiol 2014; 171(3):368-76.

27. Lee WC, Chavez YE, Baker T, Luce BR. Economic burden of heart failure: a summary of recent literature. Heart Lung 2004; 33(6):362-71.

28. McMurray J, Davie A. The pharmacoeconomics of ACE inhibitors in chronic heart failure. Pharmacoeconomics 1996; 9(3):188-97.

\section{Apéndice \\ ULS y representantes participantes}

R. Roni (Trento), A. De Marco (Belluno), M. Cecchetto (Bassano), MP Cariolato (Ovest Vicentino), A. Maroni (Vicenza), D. Maccari (Pieve di Soligo), R. Callegari (Asolo), M. Ferrari (San Donà di Piave), S. Zardo (Venezia), L. Cordella (Mirano), B. Pari (Chioggia), A. Pedrini (Alta Padovana), A. Grion (Padova), MG Zogno (Monselice), A. Ferrarese (Rovigo), V. Lolli (Adria), L. Mezzalira (Verona), S. Brasola (Legnago), L. Castellani (Bussolengo), D. Garibaldi (Lucca), P. Batacchi (Firenze), M. Rais (Viareggio), G. Riccioni (Roma A), R. Di Turi (Roma D), C. De Matthaeis (Roma F), A. Orsini (Teramo), G. La Bella (Napoli 1), E. Nava (Napoli 3), R. Moscogiuri (Taranto) 